\title{
Modelación dialéctica de la estadística desde el enfoque histórico cultural: El objeto de estudio y su medición
}

Dialectic modeling of statistics from a historical cultural approach: the object of study and its measurement

\author{
Volumen 19, Número 2 \\ Mayo-Agosto \\ pp. 1-27
}

Roy Umaña Carrillo

\section{Cite este documento así}

Umaña Carrillo, Roy. (2019). Modelación dialéctica de la estadística desde el enfoque histórico cultural: El objeto de estudio y su medición. Revista Actualidades Investigativas en Educación, 19(2), 1-27. Doi. 10.15517/aie.v19i2.37069 


\section{Modelación dialéctica de la estadística desde el enfoque histórico cultural: El objeto de estudio y su medición}

Dialectic modeling of statistics from a historical cultural approach: the object of study and its measurement

\section{Roy Umaña Carrillo}

Resumen: En este ensayo se propone la modelación dialéctica de los conceptos de la estadística en perspectiva histórico genética fundamentada en el método de ascenso de lo abstracto a lo concreto. La finalidad del trabajo es orientar el proceso de enseñanza-aprendizaje de los conceptos estadísticos en los cursos introductorios de diversas carreras universitarias y en cursos de actualización, de manera que se supere la forma generalmente superficial, fraccionada y ahistórica como se presentan las abstracciones formales en los libros de texto. Se logró otorgarle unidad a lo diverso en las dos dimensiones: el objeto de estudio, en su parte fenoménica y en los fundamentos de las escalas de medición y a su vez, enriquecer con más determinaciones los diferentes conceptos particulares según sus constantes y variantes. Por aspectos de espacio, no se incluyó la forma en que lidia la estadística con sus fenómenos particulares, pues esta se reserva para una futura entrega.

Palabras clave: lógica, enseñanza de la estadística, enfoque histórico cultural.

Abstract: This essay proposes a dialectic modeling of the concepts of statistics in a historical genetic perspective based on the method of ascending from the abstract to the concrete. The purpose of this work is to guide the teaching and learning process of the statistical concepts in the introductory courses from various university careers and trainings classes, so that the generally superficial, spread and ahistorical way of presenting the formal abstractions in the textbooks is overcome. It was possible to give unity to the diverse in two dimensions: the object of study in its phenomena part, and in the fundamentals of measurement scales, and at the same time, enrich with more determination the different and concepts serving its constants and variants. Due to space, the way in which statistic deals with phenomena was not included, and it is reserved for future articles.

Key Words: logic, statistics education, historic-cultural approach.

\footnotetext{
1 Trabaja en la Vicerrectoría de Investigación de la Universidad Estatal a Distancia de Costa Rica. Licenciado en Sociología de la Universidad de Costa Rica.
}

Dirección electrónica: rumanacarrillo66@gmail.com; rumana@uned.ac.cr

Ensayo recibido: 16 de octubre, 2018

Enviado a corrección: 5 de marzo, 2019

Aprobado: 29 de abril, 2019 


\section{Una situación problemática}

Desde una perspectiva histórica, Chaves (2007) señala que la estadística apenas se introdujo en primaria y secundaria en 1995, y constituía un tema desvinculado de la matemática y de las restantes asignaturas. A su vez, quienes enseñan carecían de una formación sólida, conceptual y pedagógica para la enseñanza de la estadística, por lo tanto presentaban los contenidos estadísticos de una forma mecánica y quien aprende se veía limitado a actuar pasivamente. Es hasta el año el año 2012 que se le da un nuevo impulso a la enseñanza de la estadística en escuelas y colegios (Ministerio de Educación Pública, 2012), cuando empiezan los procesos de capacitación a profesores del 2012 al 2014 (Gamboa, comunicación personal, 5 junio, 2013) y con un enfoque constructivista mediante la solución de problemas (Chaves, comunicación personal, 12 julio, 2013).Por lo tanto, en Costa Rica, una gran cantidad de personas ha tenido su primer contacto con la estadística en cursos introductorios ofrecidos durante sus estudios universitarios.

En las universidades, los cursos de estadística tienen altas tasas de fracaso y abandono. Por ejemplo, en la asignatura Estadística Aplicada a la Educación de la Escuela de Educación de la Universidad Estatal a Distancia de Costa Rica (UNED), de los años 1996 a 1999, la reprobación promedio fue de $30,1 \%$ y el abandono de $27,7 \%$; la suma de los dos anteriores, es decir, el fracaso total promedio fue de $57,4 \%$, con un mínimo de $51 \%$ y un máximo de 69\% (Chaves, 2000). Para el año 2004, el fracaso total es de 45,4\% (Herrera, 2005). Resultados similares se obtienen en la Escuela de Administración de la UNED, para el año 2014 (los tres cuatrimestres juntos), la reprobación y abandono del curso Estadística I fue de 51,45\%. En el curso Estadística II, sobre probabilidades, fue de 61,33\% (Sánchez, comunicación personal, 14 abril, 2015).

El estudiantado de la Escuela de Educación, en su mayoría, resuelve satisfactoriamente los contenidos que representan una bajísima dificultad: sumas, restas, multiplicaciones, divisiones y potencias elevadas al cuadrado. Sin embargo, muy pocos resuelven potencias elevadas a la 3 o fórmulas simples que implican una combinación de resta, división y potencia al cuadrado (Solano y Umaña, 2005). La mayoría de quienes aprueban el curso no pueden relacionar un nivel de medición específico de la variable y sus métodos estadísticos descriptivos apropiados, el grado de comprensión de los contenidos es muy limitado, mucho peor es la comprensión de los modelos estocásticos (Bermúdez, comunicación personal, 21 setiembre, 2012). 
La estadística, al ser una rama de la matemática, se asocia al problema de la enseñanza de esta última, que persiste y se hace evidente en los casos que se mencionarán a continuación. En la Universidad Nacional, durante el primer periodo del 2014, el 44\% de las personas matriculadas en Matemática General perdió el curso mientras que en el curso de Cálculo I, el 49\% reprobó y solo un 18\% aprobó (Alvarado, 2015). En las pruebas del Programa para la Evaluación Internacional de los Estudiantes PISA, (por sus siglas en inglés), realizadas en el 2009, en el área de matemáticas, un 84,5\% del estudiantado se ubicaron en los niveles de 0 a 2 (Montero, Rojas, Rodino, y Zamora, 2012), mientras que en el 2012, el 60\% del estudiantado costarricense mostró dominio de los niveles 1 y 2 (Mora, 2013). El nivel 2 es el nivel básico de habilidades, cuyo dominio le permitirá al estudiantado participar eficazmente en la vida productiva (Del Valle y Fernández, 2013).

A su vez, las evaluaciones realizadas por el Ministerio de Educación Pública al profesorado de secundaria del área de matemáticas arrojaron que solo la mitad de ellos se ubican en el nivel óptimo requerido para ejercer como docentes de la disciplina (Dirección de Gestión y Evaluación de la Calidad, 2010). En el caso de la estadística, los trabajos que intentan solucionar este problema no trascienden lo particular como en los casos de Enrique (2016); Pérez, (2006); Quintana, (2010), así, no se han encontrado propuestas sistémicas ya sean del tipo estructural funcional o histórico genético (dialéctico), como modos de estructuración de los conocimientos desde la totalidad. A su vez, los libros utilizados para cursos introductorios de estadística en diferentes carreras se caracterizan, de forma general, por estructurar los contenidos de una forma empírica, superficial y fraccionada, lo que se traduce en un aprendizaje con las mismas características. Esto se problematiza y se constata con detalle en la sección de resultados, además constituye la premisa que permite el ligamen con el enfoque histórico cultural (EHC) enriquecido dialécticamente.

\section{Propuesta con base en la lógica dialéctica}

De acuerdo con la situación planteada previamente se destaca la siguiente contradicción: a pesar de los esfuerzos didácticos llevados a cabo en la enseñanza de conceptos estadísticos para estudiantes de otras carreras universitarias, la orientación del proceso de enseñanza aprendizaje (PEA) propicia un aprendizaje reproductivo, superficial y poco fundamentado de esos conceptos en el estudiantado. 
Para superar dicha contradicción se planteó la siguiente tesis: la modelación dialéctica de los contenidos de aprendizaje contribuye a una mejor compresión y aplicación de los conceptos relacionados con una ciencia o disciplina en particular, en este caso de la estadística y la probabilidad, y por tanto, a la apropiación de quienes aprenden.

Resolver la contradicción implica la identificación del problema por superar como una estructuración de los conceptos que se lleva a cabo de forma empírica en el sentido sensualista o superficial, pero, al mismo tiempo, fraccionada, lo que viene a ser otra forma de empirismo. Así, desde una perspectiva dialéctica, el empirismo tiene estas dos características, que permite englobar tanto propuestas filosóficas particulares como paradigmas pedagógicos diferentes, los cuales están influenciados por las propuestas filosóficas. Para comprender los alcances de la propuesta, antes de presentar resultados es necesario explicitar la forma en que la dialéctica estructura los conceptos, en oposición a otras formas denominadas particulares que se detallan a continuación.

Durante la Edad Media, fe y razón iban de la mano, es innegable la relación estrecha que existía entre las matemáticas con la filosofía griega de Platón (Russell, 2009). Asimismo, con el método deductivo se da un desprecio por la utilidad práctica, ejemplo de ello Los elementos de Euclides (Russell, 2009). Para Platón (Pantoja, 1984), mediante los sentidos solo se puede formar una opinión subjetiva e individual: doxa. Lo real o el fundamento para la ciencia está en la razón, episteme: el mundo corpóreo y sus hechos cambiantes son percepciones que conforman una realidad inmaterial, que puede ser transmitida por medio de conceptos. Un ejemplo de idea en Platón es el gato, donde los gatos sensibles son copias imperfectas de dicha generalización (Russell, 2009). A su vez, se tiene la relación de la proposición $1+1=2$ con una manzana más una manzana (Körner, 1967). Empero, en términos de estructuración de las generalizaciones, no se ofrecen modelos.

La actividad intelectual y científica es llevada por los escolásticos entre los siglos XII y $\mathrm{XIV}$, quienes trataron de compaginar el dogma cristiano con parte del pensamiento de Aristóteles. Por lo que hay un rechazo a la experimentación empírica para demostrar o constatar cosas, y tampoco se les daba mucha importancia a las matemáticas (Barrantes y Ruiz, 1997).

En el caso de 1 es a una manzana, una interpretación sería: la manzana empírica es un miembro de la clase de las unidades de las matemáticas, así como es roja, ya que es miembro de la clase de las cosas rojas. La otra interpretación es: la manzana empírica es 
una porque se aproxima a la unidad matemática, muy próximo a Platón en este sentido, Körner (1967) se inclina por la segunda interpretación, donde abstraer o separar significa abstracción idealizante o idealización.

En relación con la estructuración de los conocimientos, el método aristotélico implica además, una especie de ordenamiento jerárquico naturalista de los conceptos: "para poder definir algo se necesitan el género y la diferencia, o, el género 'próximo' y la diferencia 'específica' [...] la esencia de las cosas viene dada por la diferencia última que caracteriza al género." (Reale, 1992, p. 142). Ejemplo de ello se tiene las taxonomías de los seres vivos, o las clasificaciones de los conocimientos de las diferentes ciencias. En el caso de la estadística se tienen las dos grandes áreas: la descriptiva y la inferencial o probabilidad, y de ahí hacia abajo se van jerarquizando los conceptos de lo general a lo particular.

Las propuestas de Platón y Aristóteles tienen las siguientes limitaciones desde un enfoque dialéctico: ambas tratan con los conceptos ya acabados, por lo que no funcionan para explicar la creación de nuevo conocimiento, solo lo anexan. Aristóteles jerarquiza el fraccionamiento de los conceptos presentes en Platón, pero sin una unidad de lo diverso, si bien esto jerarquiza, no hace más que adicionar conceptos respetando el área de conocimiento al que pertenecen. Por lo tanto, Platón y Aristóteles no proveen un sistema de acciones mentales que genere una mejor comprensión y aplicación de los conceptos, mucho menos, una forma objetiva que facilite la unidad de lo diverso, además minimizan la práctica. Es decir, no se encuentra un hilo común que le otorgue estabilidad al sistema, aspecto que se logra con modelos sistémicos, como el estructural funcional y el genético.

Kant logra una síntesis entre racionalismo y empirismo, algo que ya se aplicaba en la ciencia. Ya antes, Newton sintetizó el método científico, ello se constata en su ley gravitacional, empleando aspectos analíticos y sintéticos de forma interdependiente, el triunfo de la razón y la observación (Zeitlin, 1986). A su vez, sentó las bases sobre la forma en la que se genera el nuevo conocimiento científico y logró transformar la generalización puramente empírica en juicio teórico (en universal y necesario), sobre los límites legítimos de su objeto (Copleston, 2004; lliénkov, 1977). En las generalizaciones teóricas científicas, es decir, apriorísticas, el concepto presupone predicados que no se pueden eliminar en una experiencia futura. Al contrario, las generalizaciones empíricas (a posteriori) conservan vigencia apenas se encuentran con una experiencia que las contradice (Iliénkov, 1977). 
Desde Kant, se tiene que en la matemática pura la proposición de añadir 2 unidades a 3 unidades producen 5 unidades, es una descripción sintética y a priori, ya que está construida en el tiempo y el espacio. Las proposiciones de la matemática aplicada son a posteriori, son acerca del material empírico de la percepción, y son a priori en la medida en que son acerca de espacio y tiempo (Körner, 1967).

Asimismo, Kant lidió con las contradicciones, se centra en las categorías como principio de unión de las diferentes formas generales, y aquí empieza su dialéctica (lógica trascendental) (Iliénkov, 1977). Las contradicciones dialécticas de la metafísica anterior eran reveladas en las cuatro antinomias (tesis/antítesis) de la razón pura (Copleston, 2004). Se demostró, por tanto, que la dialéctica es una forma necesaria de la actividad intelectual para solucionar problemas sintéticos superiores, para construir una teoría que pretende la universalidad (objetividad, en términos de Kant), y no mostró una salida a los conflictos entre teorías, ideas y concepciones, salvo recomendar la regla del vive y deja vivir, mantén la propia posición y respeta la del otro (lliénkov, 1977). Es decir, a pesar de reconocer la contradicción, no logró otorgarle unidad a lo diverso, ya que siguió dentro de los límites de la lógica formal, aspecto que sí lo logró superar Hegel.

Las propuestas reseñadas son particulares o positivistas, en oposición a lo universal, justamente Adorno (1973) las denomina así por ser unilaterales y no dialécticas. Por tanto, se hace necesario profundizar esta discusión en términos de estructura, tanto de la lógica formal como de la lógica dialéctica. Eso sí, analizando la lógica no como ciencia, sino como disciplina filosófica que estudia el pensamiento lógico, su estructura conceptual de manera integral y las formas externas de pensamiento en relación con su contenido concreto, cognoscitivo (Rodríguez Ugidos, 2001). Empero, la lógica como ciencia está determinada por la lógica como filosofía.

Por lo tanto, "los problemas de la filosofía, como ciencia especial, giran de uno u otro modo en torno a la cuestión acerca de qué es el pensamiento y cuáles son sus relaciones recíprocas con el mundo exterior" (Iliénkov, 1977, p. 84). Lo unitario es la relación entre pensamiento y realidad, este proceso se cristaliza en la formación de conceptos, hay muchas lógicas o modos de concebir dicha síntesis: lo diverso se compone de diferentes propuestas particulares: Platón, Aristóteles. Este proceso entre pensamiento y realidad se caracteriza por un nivel de profundización o complejización de invariantes, que va de lo simple a lo complejo, incluso de lo ideal a lo material. 
A nivel estructural, la lógica formal, como disciplina filosófica, no admite la contradicción, la cosa es o no es, no puede ser y no ser al mismo tiempo. Ella parte de tres principios:

1) El principio de identidad: todo objeto es idéntico a sí mismo (Kohan, 2016). A es $A$, es el principio de la tautología, la coherencia, la repetición pura y simple (Lefebvre, 1970). Ejemplo de ello: "Wittgenstein sienta las bases de su método enunciando la proposición «Los objetos forman la sustancia del mundo. De ahí que no puedan ser compuestos» (tractatus 2.021)" (Adorno, 1973, p. 55). O el globo rojo es rojo.

2) El principio de no contradicción: $A$ no es no- $A$, así se introducen en la identidad la diferencia, la relación, la contradicción, donde la diferencia y la relación se introducen como contradicción, pero no a la inversa (Lefebvre, 1970, p. 156). O el globo rojo no es un globo verde.

3) El principio del tercero excluido: para Lefebvre (1970) una afirmación no puede ser al mismo tiempo verdadera y falsa. El principio o $A$ o $-A$ (no $A$ ) implica contradicción, pero se debe tomar uno de los predicados, no admite los dos al mismo tiempo. El tercero está excluido (Kohan, 2016). O el globo es rojo o es verde, existe la posibilidad de que sea rojo y verde a la vez, pero es una adición, no una contradicción.

En cambio, la dialéctica parte de la diferencia, la contradicción como relación, sí puede ser y no ser la vez (Kohan, 2016). Utilizando la terminología de Lefebvre, la identidad se fija con dos componentes o predicados que se contradicen entre sí, es la forma intencionada con la que Hegel logra unir las contradicciones, que la lógica formal reconoce, pero no puede lidiar con ellas. Para Hegel, la verdad (concreta) es la unidad de la identidad y de la diferencia (Lefebvre, 1970, p. 157).

De ahí que Hegel denomina la lógica formal como raciocinio, que actúa de forma desglosadora y abstractiva, alcanza la solidez y la certeza de los acontecimientos, pero con la limitación de que no va más allá de la certeza inamovible y su capacidad de diferenciarse de otras certezas, es conceptuar en general (Davýdov, 1981). Al contrario, la unidad de lo diverso, lo racional, lo dialéctico, la razón es para Hegel: la forma de descubrir la autenticidad del objeto como ente concreto, como unidad de distintas definiciones, es el ente concreto, no es unidad simple y formal, sino unidad de diferentes generalizaciones (Davýdov, 1981). 
La generalización dentro de la lógica dialéctica es una unidad compleja y sobrelleva la sinonimia, es la totalidad o todo en Hegel (Pantoja, 1984), la unidad de lo diverso, conexión, integridad (Davýdov, 1981), unidad en la variedad (lliénkov, 1977); o ley, esencia de la naturaleza, de nuevo en Hegel (Davýdov, 1981); imagen del todo en Marx (Davýdov, 1981); célula, ley, forma (Marx, 1959); también en Hegel, universal concreto en contraposición a lo universal abstracto (lliénkov, 1977).

Asimismo es para Davýdov (1981) abstracción concreta, relación concreta general, célula objetiva del todo investigado, enjundiosa abstracción, o célula. Davýdov prefiere denominarla abstracción real y enjundiosa que, a diferencia de la abstracción formal, es histórica, pues constituye su base genética y su contenido existe realmente en forma de relación contemplable, y no solo en la mente; en contraposición, la abstracción formal o lo abstracto es algo simple, exento de diferencias, fragmentario y no desarrollado, parte del todo (1981), y agrupa muchos sinónimos más. En cambio Morin (2007, p. 56), en el capítulo "Unitas multiplex" (unidad compleja) afirma que "Es pertinente, en cambio, concebir una unidad que asegure y favorezca la diversidad, una diversidad que se inscriba en una unidad", pero no es una visión histórico genética. Así manifestada, no parece postular, desarrollo, superación, donde lo viejo quede contenido en lo nuevo en una nueva trama de interrelaciones.

En síntesis, las abstracciones formales unen lo común, ello permite diferenciar una cosa de otra, en cambio, la dialéctica, es la forma que desarrolló Hegel para unir lo diferente, los diferentes conceptos de una ciencia en particular, de ahí que se denomina la unidad de lo diverso o atracción enjundiosa. Y es que tanto los conceptos empíricos (o representaciones) como los científicos, estructuran lo común a cada uno de ellos, es lo que permite diferenciar una cosa de la otra. Para Hegel, ambas tienen la misma estructura (lliénkov, 1977). Esa es la riqueza del método dialéctico, que va de lo concreto a lo abstracto y se eleva de lo abstracto a lo concreto (lliénkov, 1975). Es decir, a más abstracción, mayor concreción. En otras palabras, los diferentes conceptos científicos particulares lograron el salto de lo concreto a lo abstracto, por tanto, a la dialéctica le toca elevarse de lo abstracto a lo concreto enriquecido, la dialéctica trabaja con los logros de las abstracciones formales y las enriquece, genera enjundia.

En términos de Dussel (1991, p. 48), "Lo concreto es concreto porque es la síntesis de múltiples determinaciones, por lo tanto unidad de lo múltiple. Aparece en el pensar como 
proceso de síntesis, como resultado, no como punto de partida". Ello implica que en el análisis de las diferentes abstracciones formales, estas se vayan enriqueciendo de múltiples determinaciones. Mejor dicho, en el proceso de investigación, con el fin de identificar la abstracción enjundiosa, se hace necesario aumentar e intensificar las determinaciones de las abstracciones formales, con el fin de discernir lo constante (lo invariante, la abstracción enjundiosa, la ley, el todo, histórico genético, etc.) y lo variable o particular de cada concepto formal.

De ello se colige que efectivamente los conceptos científicos no son arbitrarios, lo arbitrario es el manejo que se hace de sus determinaciones. En un PEA empírico, en términos sensualistas, se obvian muchas de sus determinaciones o fundamentos, de ahí su superficialidad. En cambio, en otros modelos donde se puede lograr una mejor comprensión de las determinaciones del concepto, no superan lo fraccionado o su manejo particular, es decir, se carece de un análisis relacional entre conceptos diferentes.

A su vez, el hecho de guiarse a través de los conceptos desde una lógica formal, no niega la dialéctica (Iliénkov, 1977). Esto, por cuanto la dialéctica es hospitalaria, la negación dialéctica no es destrucción, es todo lo contrario, es enriquecimiento, enjundia. Por lo tanto, para una mejor comprensión, es necesario diferenciar el análisis genético del estructural funcional. En este último, el objeto complejo y desarrollado se considera como una serie de subsistemas de niveles, estructurales funcionales, jerarquizados. En el dialéctico, el objeto complejo es considerado genéticamente, en el origen de las propiedades integrales del sistema por la vía del análisis de la proporción original elemental. En el primer caso, los nexos de funcionamiento actúan como formadores del sistema, en el segundo, los de desarrollo y génesis. Ambos modelos son de distinta naturaleza y generan distintos sistemas. (Salmina, Sorokin, y Chernysheva, 1984). Para estos autores (Salmina et al., 1984), incluso para Reshetova (1988), ambos modelos son sistémicos. Después de la explicación de lo que es la dialéctica queda por dilucidar su conexión con el EHC.

\section{Lógica dialéctica y el EHC}

La dialéctica en Vygotsky (1934) se observa claramente en el método, en el análisis de unidades versus el análisis de elementos, que conserva todas las propiedades básicas del total y no puede ser dividida sin perderlas; por ejemplo, la verdadera unidad de análisis de la biología es la célula, ya que posee todas las propiedades básicas del organismo vivo. Esta 
definición es un buen ejemplo de la relación entre la parte y el todo, pero más allá de una visión sistémica, lo importante en dichas definiciones es el movimiento de una ciencia que permite captar las constantes y variantes en las diferentes abstracciones formales. A su vez, Vygotsky apuesta por el método de Marx utilizado en El Capital de forma explícita (2000). En Vygotsky (1934) y en Davýdov (1981), en el pseudoconcepto (complejo) y en el concepto subyace una generalización del mismo tipo, un mismo contenido, pero por diferentes operaciones intelectuales. Es la misma conclusión a la que llega Hegel (supracitado) sobre las representaciones y los conceptos científicos en relación con su contenido.

Ahora, ¿por qué Davýdov? Pues, si bien Vygotsky aplicó la dialéctica materialista para el desarrollo de la psicología (Vygotski, 2000), consiguiendo sentar sus bases teóricas y metodológicas con múltiples aportes con base en unidades complejas: análisis de unidades, ley evolutiva, zona de desarrollo próximo, etc.; en el caso de la modelación de los contenidos para direccionar el aprendizaje logró desarrollos de carácter sistémico y jerárquico sin embargo no alcanzó profundidad en este aspecto, lo que permitió a Davýdov ampliarlo mediante la modelación dialéctica de los contenidos.

Después de diferentes aproximaciones al problema de la estructuración de los contenidos, Vygotsky plantea la diferencia entre conceptos formales (habituales 0 espontáneos) y los auténticamente científicos. En los primeros, el camino transcurre de lo concreto a lo abstracto; en el segundo grupo, de lo abstracto a lo concreto, del concepto a la cosa. Para la enseñanza de conceptos científicos, se establecen dependencias entre conceptos, la formación de un sistema con estos, la concienciación de la propia actividad mental, y una relación especial del sujeto con el objeto que permite reflejar en él el internamiento en la esencia del objeto (Davýdov, 1981). Esto desemboca en la pirámide de conceptos, un sistema donde el proceso complejo de pensamiento va de lo general a lo particular y de lo particular a lo general casi simultáneamente. Dicha pirámide es jerárquica, no es asociativa ni estructural de las imágenes perceptibles o representables (Davýdov, 1981).

A partir de este punto, para Vygotsky, el problema corresponde a cómo diferenciar los conceptos discursivos empíricos o representaciones generales, y los conceptos genuinamente científicos, pues lo fundamental radica en que los conceptos verdaderamente científicos se adquieren con el desarrollo y con la ayuda de quienes enseñan, quien elabora un sistema con estos y se le transmite al estudiantado. En cambio, los conceptos habituales 
o usuales se desarrollan en forma extrasistémica, relacionándose con el medio social a través de su experiencia personal (Davýdov, 1981). Con la salvedad de que los conceptos cotidianos (sincréticos, en complejo, y pseudoconceptos), no desaparecen, sino que constituyen la base de los conceptos reales o científicos.

Empero, para Davýdov (1981), Vygotsky no hace una fundamentación circunstanciada de dicha diferenciación, ya que no se basa en una diferencia del contenido objetivo de la formación de los conceptos, sino en el método, en las vías de asimilación (proceso de estudio sistematizado por el profesorado en la escuela, versus la experiencia personal del estudiantado, proceso espontáneo). A su vez, aclara Davýdov (1981), que los conceptos habituales o empíricos también poseen un determinado sistema (poseen dependencias genérico-específicas), y que igualmente se adquieren en la escuela, sobre todo en primaria. Vygotsky omite desarrollar que realmente los conceptos científicos sí tienen un sistema especial: "El asimilar partiendo 'de lo general', de la definición discursiva no se caracteriza en sí mismo por la entraña científica del concepto: de modo análogo pueden presentarse en el estudio cualesquiera nociones generales cotidianas, empíricas." (Davýdov, 1981, pp. 226227).

En suma, a Vygotsky no le alcanzó su corta vida para llegar a estructurar de forma dialéctica los conceptos por aprender, que pareciera ser el paso lógico a seguir, de ahí la importancia de su seguidor Davýdov. Ahora queda por precisar lo empírico en el aprendizaje dentro del EHC, desde la perspectiva que se ha desarrollado en esta propuesta. Para Davýdod (1981), los diferentes modelos no dialécticos de orientación del PEA, sean explícitos o no de la lógica con que operan, se consideran como empíricos, ya que todos ellos desarrollan perspectivas fraccionadas y particulares (positivistas), y esto es explicado por la unión de lo común: la abstracción formal tiene un carácter fraccionado y ahistórico (Davýdov, 1981).

Sin embargo, tampoco se descarta una enseñanza superficial donde primen las operaciones, los cálculos, y no la comprensión del concepto, o por el contrario, se anime más a la memorización de conceptos y no su aplicación, o una mezcla de ambos. Por tanto, se incluyen aquí, como empírico, tanto lo superficial como lo fraccionado, acogiendo tanto PEAs sensualistas como perspectivas de enseñanza-aprendizaje consideradas constructivistas y que hacen un uso explícito de la lógica formal como ciencia: como en los casos de Piaget (Davýdov, 1981) y Bruner (2010), esto es así ya que en ambas posibilidades se hace una 
modelación de los contenidos de forma fraccionada y ahistórica. Para el caso de lo que denomina Canfux (2000) como educación tradicional, retrata más un PEA empírico en sentido sensualista. Aunque en algún momento hace alusión también a la falta de un carácter integrador de los conocimientos.

EI EHC, desde la lógica dialéctica, ataca y supera el fraccionamiento y la ahistoricidad del tratamiento de los contenidos de aprendizaje científicos en su fundamento lógico formal, así como los que se llevan a cabo de forma superficial. Irigoyen (2013), tipifica muy bien este fraccionamiento de los contenidos de aprendizaje, el saber se desmenuza en múltiples paquetes autónomos, entiende la educación como un producto industrial, así el trabajo del estudiantado es una suma de pequeños trabajos en fragmentos requeridos en las distintas asignaturas/producto, que ocupa todo su tiempo, instaurándose la redundancia y repetición sin unidad, ello dificulta que el estudiantado se apropie de lo que aprende, y no queda espacio ni energía para la construcción de un núcleo duro, totalizante. Por supuesto, la caracterización anterior incluye las estructuraciones que se hacen de diferentes ciencias del tipo aristotélico comentadas anteriormente. A continuación, se ejemplifican algunos procesos de enseñanza aprendizaje siguiendo el hilo conductor desarrollado hasta el momento.

Una didáctica con una lógica explícita se encuentra en Amos Comenio, quien opone la lógica aristotélica a la platónica como forma de modelar los contenidos de aprendizaje: "[...] que casi nunca han sido enseñadas las artes y las ciencias de un modo enciclopédico, sino fragmentariamente [...] como montones de maderos o de sarmientos, en los que nadie advierte la razón en virtud de la cual están unidos" (Comenio, 2002, p. 95). Empero, ya se reseñó su carácter aditivo. Por su parte, en el conductismo se promueve el aprendizaje repetitivo (rote learning) y la importancia del algoritmo, donde la generalización se logra mediante la transferencia de una situación a otra de forma empírica, mediante repeticiones (estímulos) (Ertmer y Newby, 2013), el sistema de acciones se limita a ello, por lo que no hay una estructuración de los conceptos, es decir, una unidad de lo diverso.

La lógica formal, como ciencia, es explícita en los casos de Bruner (2010) y de Piaget (Davýdov, 1981), el concepto de reversibilidad de Piaget es importante para Davýdov (1981), al punto de considerarlo una categoría sistémica, lo cual propicia el descentramiento de la relación del sujeto con el objeto, permitiéndole al primero destacar y consolidar las peculiaridades de la invariabilidad del objeto que subsiste de modo estable entre sus variadas transformaciones particulares. No obstante, no revela los fundamentos de los 
tránsitos que llevan de las acciones a las operaciones (Davýdov, 1981). Además, al no superar los supuestos de la lógica formal, no propone una metodología que le brinde unidad a lo diverso.

Los PEA ejemplificados anteriormente, sin pretender ser una lista exhaustiva tienen en común que no superan el carácter fraccionado y ahistórico de los contenidos, ya que parten de los postulados de la lógica formal.

\section{Resultados de la aplicación del modelo}

¿Qué es la estadística? En los libros utilizados en el PEA de estadística en cursos introductorios de diversas carreras universitarias, la definición de estadística se lleva a cabo de una forma empírica. Así, se define como una "Ciencia que recoge, organiza, presenta, analiza e interpreta datos con el fin de propiciar una toma de decisiones más eficaz." (Lind, Marshall y Wathen, 2012, pp. 4-5). En general, los elementos presentes en dicha definición como recoger, organizar, analizar e interpretar, se repiten en textos similares, (Burgos García y Burgos Gómez, 2016; Burgos Gómez, 2016; Daniel, 2008; Gómez Barrantes, 2012; Hernández, 2010, 2012; Juan, de la Fuente y Vila, 2011; Juárez Duarte, Ylé Martínez, Flórez Arco e Inzunsa Cázares, 2012; Mendenhall, Beaver, y Beaver, 2010; Pérez y Romero, s. f.; Salama, 2002; Spiegel y Stephen, 2009; Triola, 2004, 2013). Asimismo, dichas definiciones tienen la particularidad de no esclarecer el objeto de estudio de la estadística en su parte fenoménica, tampoco aclaran sobre los modelos teóricos que le son propios para lidiar con sus fenómenos, y que le otorgan su especificidad. Ese estilo de definir las ciencias es apropiado si se trata de ocupaciones manuales. En adición, en los libros supracitados, en relación con el tema de los datos, y la variabilidad de estos, tampoco se supera su presentación empírica, superficial, o bien, hay que suponer su variabilidad. Como excepción se tiene a Salama (2002) y Juárez et al. (2012), quienes usan el término indeterminismo en lugar de variabilidad, sin más profundización o carácter relacional.

Por su parte, Juárez et al. (2012) sí plantean la diferencia entre una pregunta determinista y una estadística, y no emplean el término indeterminista. Walpole, Myers, Myers, y Ye (2012) utilizan los términos variación, incertidumbre y variabilidad, pero no se refieren a los fenómenos deterministas. Verdoy, Beltrán, y Peris (2015) se refieren a la ciencia que se encarga de fenómenos de tipo aleatorio. Así como otros se refieren a la incertidumbre y a la aleatoriedad (García del Valle, 1995; Montanero Fernández y Minuesa 
Abril, 2018; Zieffler y Catalysts for Change, 2013). Solo Morettin (2010) utiliza la dicotomía determinista e indeterminista. Sin embargo, ninguno de los libros anteriormente mencionados plantea un carácter relacional entre los fenómenos, lo que no ofrece mayor claridad sobre el campo de la estadística. Por eso, se identifican estas definiciones como empíricas, superficiales, sensualistas, además de que son presentadas de forma fraccionada.

Girón (1999), a diferencia de los autores supracitados, sí ofrece definiciones, por ejemplo la incertidumbre se refiere a fenómenos no conocidos o no controlables y azar se refiere a los que no se pueden predecir pero se conocen sus probabilidades, como con el lanzamiento de una moneda. Aunque en el caso de la incertidumbre se pueden utilizar probabilidades no necesariamente uniformes, es decir personales o subjetivas. De lo expuesto por Girón, interesa resaltar que azar e incertidumbre, ambos se caracterizan por no tener certeza absoluta, o determinismo. Asimismo, esto quiere decir que no hay control sobre las causas, o bien, en muchos casos son desconocidas. Por su parte, Viertl (2003) introduce la incertidumbre por medio de la simpleza de los modelos lineales versus las complejidades del sistema, mientras que para Prigogine (1977), la incertidumbre se genera por medio de la entropía. Álvarez y Álvarez (2004) ofrecen un modelo sistémico de los fenómenos, el cual es bastante esclarecedor sobre los tipos de fenómenos que se toman en cuenta en matemáticas. Sin embargo, en dicho modelo no se explicita cómo se transita del determinismo al indeterminismo, o viceversa. Ahora, es necesario preguntarse ¿qué determinaciones tiene la incertidumbre?, denominada empíricamente variabilidad de los datos.

Para enriquecer dicho concepto, primero se ha identificado como ley o célula la relación entre los fenómenos deterministas e indeterministas, similar al anverso y al reverso de una moneda. Ello lo expone magistralmente Rao (1997, p. 34), quien manifiesta:

La vida sería intolerable si los fenómenos ocurrieran al azar, de una forma completamente impredecible, y carecería de interés si, en el otro extremo, todo fuera determinista y completamente predecible. Cada fenómeno es una curiosa mezcla de ambos, que "hace la vida más complicada, pero no carente de interés", como J. Neyman solía decir.

La relación anterior permitió elaborar una definición más acogedora de la variabilidad de los datos (o de la incertidumbre), que muestra esta unidad de contrarios. Se comprende 
por incertidumbre una colección de datos, cada uno de ellos en sí determinista que varía por azar. Lo anterior se debe a que las explicaciones sistémicas, pero no genéticas de Viertl y Prigogine comentadas más arriba, si bien reconocen los dos fenómenos deterministas e indeterministas a la vez, no dejan de estar separados, continúan teniendo carácter dual, parafraseando a Hegel, no se han fusionado como una unidad de la identidad y la diferencia.

Por ejemplo, si en un grupo de personas se le pregunta a sus participantes la edad cumplida, cada uno de esos datos es un determinismo, pero juntos se indeterminan. Así sucede con otros fenómenos, la muerte es un determinismo, pero las muertes ocurren en diferentes momentos, así se indeterminan. Como ejemplifica Girón (1999), antes de la invención de los computadores no se tenía claro cuál era el decimal de pi en la posición 9997, por lo que solo se tenía la certeza de que hay una probabilidad de 0,5 de que fuera par. Sin embargo, con el advenimiento de las computadoras se determinó que el valor de pi en dicha posición es 5. Ahora si se preguntara qué número ocupa la posición 10000000000000000000099954345671089 , se debería volver al modelo probabilístico propuesto, reducir la incertidumbre a la probabilidad de 0,5 de que el dígito sea un número par. Girón basa su modelo probabilístico para reducir la incertidumbre con base en la ley de los grandes números, conforme crece el número de experimentos repetidos, se lleva a cabo la estabilidad de la frecuencia de los diferentes eventos.

Otro ejemplo de un fenómeno que muestra sus dos caras, determinista e indeterminista, se relaciona con la gravedad. Aristóteles pensaba que los objetos más pesados caían más rápido que los objetos más livianos, eso es fácil observar si se compara la caída de una hoja de papel con la caída de una moneda, o este último con la caída de una pluma. Galileo, de forma experimental, demostró que los objetos con diferente peso caen con la misma aceleración. Sin embargo, no explicó el porqué de ello (Wilson, Buffa, y Lou, 2006). Newton sí lo hizo, mediante su segunda ley de movimiento, donde se relacionan aceleración, fuerza y masa (Wilson et al., 2006). Con esta fórmula $t=\sqrt{2 d / g}$, se puede calcular el tiempo de caída de un objeto en una distancia determinada, donde $d$ es la distancia recorrida y $g$ la constante gravitacional de 9,8 metros por $s^{2}$; así, si se lanza una piedra a una altura de 5 metros, esta tarda en llegar al suelo 1,01 segundos (Wilson et al., 2006).

Si se realiza el experimento de lanzar piedras a 5 metros de altura del suelo y comenzamos a anotar los tiempos de caída, en 10 lanzamientos se pueden obtener los 
siguientes resultados en segundos obtenidos con base en un simulador al lanzar una piedra a 5 metros de altura: 0,92 (1), 0,94 (2), 0,98 (1), 0,99 (2), 1,0 (1), 1,04 (1), 1,07 (1), 1,09 (1) (Programa de lanzamiento de piedras, 2013). Los números entre paréntesis representan la frecuencia de las medidas. Esta variabilidad se debe a que las leyes describen una situación ideal, en cambio, en un experimento se trata de controlar varios aspectos del fenómeno, tales como las corrientes de aire o el error humano a la hora de hacer las medidas de tiempo.

También se tiene la incertidumbre generada a partir del uso de instrumentos de medición. La metrología parte del hecho de que las mediciones no son exactas, lo que genera una variabilidad que posibilita el uso de la estadística, pues de previo admite la incertidumbre al realizar las mediciones. La incertidumbre es un "Parámetro, asociado al resultado de una medición, que caracteriza la dispersión de los valores que, con fundamento, pueden ser atribuidos al mensurando" (Marbán y Pallecer, 2002, p. 24). Por ejemplo, para Baird (1991) las medidas no son exactas, como en el simple caso de medir la longitud de un cuaderno con una regla, sino que es una aproximación, se añade incertidumbre a la medida y, por tanto, variabilidad a las mediciones, por lo que no se estaría buscando una medida exacta, sino un rango o intervalo aceptable para dicha medida.

Un enfoque como el anterior entra en contradicción con planteamientos como el de Triola (2013, p. 5) al defender que "Para aprender a pensar en términos estadísticos, suelen ser más importantes el sentido común y las consideraciones prácticas que la aplicación irreflexiva de fórmulas y cálculos." Dicha posición es bastante empírica en términos sensualistas, empero, se demostró que se puede enriquecer la compresión del tema en cuestión fundamentándolo. Además, el sentido común remite a la experiencia individual, por lo cual, no es capaz de sustituir por sí sola conceptos científicos, ese no es el objetivo de una educación científica.

En suma, el objeto de estudio de la estadística es la incertidumbre, y se concibe como una colección de datos cada uno de ellos en sí determinista que se indeterminan por azar. Las ciencias se deslizan por un tránsito entre determinismos e indeterminismos y viceversa, y en varias ocasiones solo es posible aproximarse de forma indeterminada a los fenómenos, de ahí la ubicuidad de la estadística en las diferentes ciencias. Ahora, es necesario responder a la pregunta ¿cómo se miden los fenómenos?

Esta discusión se presenta en los libros de estadística para no estadísticos de una forma bastante superficial y fraccionada, ya que se suelen presentar ciertas características 
de las escalas, sin sus fundamentos, y mucho menos se encontró un carácter relacional entre dichas características (Burgos García y Burgos Gómez, 2016; Burgos Gómez, 2016; Daniel, 2008; Gómez, 2008, 2012; Hernández, 2012; Johnson, 1990; Lind, Marshall, y Wathen, 2012; Lipschutz y Schiller, 2000; Mendenhall, Beaver, y Beaver, 2010; Quintana, 1983, 1989; Triola, 2004). Asimismo, la exposición de contenidos es ahistórica, al menos Saldkind (1999), apunta a Stevens como el autor de los diferentes niveles de medición en escalas: nominal, ordinal, intervalo y razón, pero sin exponer sus fundamentos.

En relación con la acumulación de características entre las diferentes escalas propuesta por Stevens $(1946,1959)$, pocos autores se refieren a ellas, pero de una forma no fundamentada (Hernández, 2012; Lind et al., 2012; Salkind, 1999; Triola, 2004), mucho menos se hace referencia a las propiedades axiomáticas de las escalas explicadas por Guerra, Correa, Núñez, y Scarón (1994) y Díez (2009). Tampoco se refieren a las transformaciones invariantes de las escalas de Stevens $(1946,1959)$, ni los libros para la enseñanza de la estadística citados en esta sección ofrecen una explicación de por qué se pueden hacer frecuencias en todos los niveles escalares, por qué sí se puede calcular una mediana en las escalas: ordinal, intervalo y de razón, pero no en la nominal, y por qué se aplican el promedio y la desviación estándar en las escalas de intervalo y razón, y no en la escala nominal ni en la ordinal.

Incluso se encuentran posiciones extremas, por ejemplo, se afirma que en el nivel nominal se cuenta, pero no se mide (Burgos, 2016). Asociado a ello, se tiene la posición de Diez (2009), quien elimina de tajo el nivel nominal, esto porque su concepto unificador es la gradualidad de las escalas, por tanto solo se fija en los niveles ordinal, de intervalo y de razón. No obstante, sí incluye la nominal en escritos anteriores (Díez, 1997a, 1997b).

La perspectiva teórica escogida para determinar por qué podemos medir las cosas trasciende lo matemático sin anularlo (Krantz, Luce, Suppes, y Tversky, 2007), es decir, se sobrepasa lo disciplinar y lo multidisciplinar, por tanto se necesita relacionar diferentes ciencias, como las física, la filosofía, la psicología y la estadística, incluso hay cabida para la historia y hasta para la sociología.

Con el fin de identificar la abstracción enjundiosa que le otorgue unidad a lo diverso a las escalas de medición, se identificó la relación de lo cualitativo con lo cuantitativo por medio de patrones específicos a cada nivel escalar. 
En un primer momento se pensó en denominarlo como la relación entre lo cualitativo con lo cuantitativo, pero esta acepción ejemplifica mejor la matemática como un todo, ya que es la ciencia que se distingue por cuantificar lo cualitativo, ya sea en su forma pura o aplicada. Stevens (1959, p. 394), afirma que la medición "Consiste en asignar números a los objetos o sucesos de acuerdo con cierta regla". No obstante, se considera que es un nivel general que tampoco aclara lo específico de la medición, ya que bien podría referirse a la matemática aplicada. Las escalas de medición permiten relacionar el mundo sensible con el mundo de los números.

De manera similar a Álvarez y Álvarez (2004), Davýdov (1981) se refiere a la medición de una magnitud como sigue: $A / C=N$, para $A$ = cualquier objeto planteado como magnitud, $C=$ cualquier medida y $N=$ cualquier número.

Lo anterior está bien para ese nivel específico, sin embargo, en este trabajo con el fin de hacer un planteamiento que involucre las diferentes escalas de medición, la relación anterior se representa como $C / P=N$, donde $C$ es igual a una cualidad de los objetos en cualquier nivel escalar, $P$ es cualquier patrón correspondiente a cualquier nivel escalar, y $N$ cualquier número, ya sea a nivel nominal, ordinal, cardinal, o en la escala de los números reales. Así, cada escala relaciona lo cualitativo con lo cuantitativo de una forma específica siguiendo un patrón determinado, por lo tanto relaciona el mundo sensible con el mundo de los números.

Lo anterior permite incluir el nivel nominal como Cualidad/Clasificación = Número nominal. Se considera que la escala nominal no se puede eliminar, ya que es un paso previo para llegar a la gradualidad, una vez realizados los conteos. De hecho, en todos los niveles escalares se pueden realizar conteos o se pueden llevar a la escala absoluta. Esta última escala permite generar afirmaciones como: hay más hombres que mujeres o la población de la provincia de San José es más grande que la de Puntarenas (Luce y Suppes, 2002). Aquí, incluso, se está incluyendo la variable cuantitativa discreta.

El nivel de relación entre las características de los objetos y las características de los números es posible gracias a los morfismos e isomorfismos, que se forman entre ellas, y ello se explica mediante los desarrollos teóricos en el nivel de propiedades axiomáticas (Guerra et al., 1994; Díez, 2009), y la propuesta de Stevens (1946, 1959) sobre las transformaciones invariantes de las escalas mediante funciones. Por ejemplo, en la escala de razón, así como hay un isomorfismo cuando se transforman los metros a centímetros y de kilos a gramos, o, 
al contrario, gracias a la operación inversa, también se puede reproducir ello en el objeto, un kilo de arroz se puede descomponer en gramos, después se pueden juntar y convertirse de nuevo en un kilo, un isomorfismo entre las propiedades de los números y las propiedades de los objetos.

Un ejemplo de morfismo es el que se genera entre los axiomas de orden en el nivel de las magnitudes, que viene a ser una especie de orden débil, por ejemplo, se puede sostener que el objeto $A$ es más pesado que el objeto $B$ y este más pesado que el objeto $C$, por tanto, $A$ es más pesado que $C$ (transitividad); pero no se puede aplicar ese razonamiento a una afirmación tan fuerte como $A$ es un kilo más pesado que $B$, y este es un kilo más pesado que $C$; por tanto, $A$ es un kilo más pesado que $C$, ya que $A$ es en realidad dos kilos más pesado que $C$ (Guerra et al., 1994). De ahí que Luce (1996) lo denomine un orden débil. Y esto ocurre debido a que los axiomas se expresan en el nivel ordinal. Las escalas que presentan orden son asimétricas y transitivas, la nominal es simétrica y transitiva pero en términos de diferencias (Guerra et al., 1994), ya que las categorías en este nivel sirven para diferenciar los atributos de los fenomenos: mujer/hombre, sí/no, globo rojo/globo verde, etc. Así la simetría se ejemplifica como: el globo rojo es diferente del verde, así como el verde es diferente del rojo y hay transitividad cuando se tienen tres elementos diferentes en la comparación.

También con base en los planteamientos de Stevens y las propiedades axiomáticas citados, en lo referente tanto a las transformaciones invariantes de las escalas como a la acumulación de características en ellas, todo ello permite generar una regla para la asignación de los estadísticos descriptivos con su respectivo nivel escalar. Para ilustrar, en las escalas nominal, ordinal, intervalo y razón, en todas se pueden hacer frecuencias y calcular la moda debido a que acumulan la propiedad de clasificación de la nominal, en otras palabras, en todas ellas se puede contar, se llevan a la escala absoluta. Una vez que se terminan los conteos en la escala nominal, se pueden aplicar cálculos como razones, proporciones y porcentajes. El orden, entendido como asimétrico y transitivo, presente en las escalas: ordinal, intervalo y razón, explica por qué sí se puede aplicar la mediana en ellas y no en la nominal. Y las propiedades aditivas explican por qué se puede aplicar el promedio y la desviación estándar en las escalas de intervalo y razón, y por qué no se pueden aplicar en la nominal y la ordinal. 
Esta discusión puede extenderse por múltiples aristas, como la explicación sobre las características aditivas de la temperatura medida en grados centígrados y Fahrenheit, pero no tiene todas las determinaciones que tiene la escala de razón, o la transformación de la temperatura a nivel de intervalo a una magnitud en grados Kelvin. Por otro lado, las escalas absoluta y relativa, incluso la probabilidad, tienen propiedades aditivas, pero no se puede decir que sean extensivas como las magnitudes, de ahí la importancia de Patrick Suppes (Díez, 2009), que une las propuestas axiomáticas desarrolladas por Helmholtz, Hölder y Campbell, junto con las transformaciones invariantes de las escalas de Stevens ya comentadas.

La teoría de las invariantes de las escalas de Stevens es apoyada por unos pero también tiene sus detractores, lo mismo acontece con la medición de aspectos subjetivos del ser humano de forma cuantitativa (Díez, 2009; Narens y Luce, 1993). Savage y Ehrlich (1991) aclaran que muchos campos de la teoría de la medición no están exentos de contradicciones. Lo anterior demuestra el carácter no acabado de esta teoría.

\section{A modo de cierre}

Se identificó una abstracción enjundiosa para los fenómenos de estudio de las diferentes ciencias. La relación entre el determinismo y el indeterminismo tiene la característica de ser irreductible, ya que engloba todas las manifestaciones posibles en ese nivel establecido, al punto que se logró una explicación de la variabilidad de los datos. Al mismo tiempo, tiene la bondad de que se aplica a procesos simples y complejos, por lo que genera estabilidad y objetividad en el sentido de invariabilidad a todas las manifestaciones de los fenómenos involucrados ya que la construcción de la incertidumbre se genera de múltiples certidumbres.

Asimismo, se logró la unidad de lo diverso con las escalas de medición, y se identificaron una gran cantidad de determinaciones no tomadas en cuenta en los textos utilizados en el PEA de la estadística. Se identificó una regla para la asignación de estadísticos descriptivos con sus respectivas escalas. Igualmente, para medir los fenómenos deterministas e indeterministas se utilizaron las mismas escalas, parece ser obvio, pero esto no se aclara en ninguno de los textos consultados.

Lo anterior es consecuencia de la aplicación del método de ascenso de lo abstracto a lo concreto, que permite tanto otorgarle un carácter de totalidad a una ciencia en particular, 
así como enriquecer de más determinaciones los diferentes conceptos particulares. En otras palabras, identificar lo constante y lo variante en los diferentes conceptos. Esto contribuye a la apropiación de los conocimientos en quienes aprenden. Si bien las abstracciones enjundiosas identificadas son dialécticas, al ser propuestas, no dejan de ser generalizaciones empíricas o a posteriori, bien podría encontrarse con una experiencia que las contradiga. Queda por desarrollar los modelos que utiliza la estadística para enfrentar los fenómenos indeterministas, así como falta por mostrar los resultados de dicha modelación dialéctica en un PEA, por razones de espacio esto se tratará en futuras entregas.

\section{Referencias}

Adorno, Theodor Ludwig Wiesengrund. (1973). La disputa del positivismo en la sociología alemana. Barcelona-México D. F.: Ediciones Grijalbo.

Alvarado, Josué. (16 de agosto de 2015). ¿Poca preparación en el colegio? Mayoría de estudiantes de la UNA pierden cursos de matemática elemental; MEP rechaza responsabilidad. Crhoy.Com, Recuperado de http://www.crhoy.com/mayoria-deestudiantes-de-la-una-pierden-cursos-de-matematica-elemental-mep-rechazaresponsabilidad/

Álvarez, Evelyn, y Álvarez de Zayas, Carlos. (2004). Elementos epistemológicos de la matemática y su enseñanza. Bolivia: Grupo Editorial Kipus.

Baird, D. C. (1991). Experimentación. Una Introducción a la teoría de las mediciones y al diseño de experimentos (2a. ed.). México: Pearson Educación.

Barrantes Campos, Hugo, y Ruiz Zúñiga, Ángel. (1997). Elementos de cálculo diferencial. San José, C.R.: Editorial de la Universidad de Costa Rica.

Bruner, Jerome. (2010). Realidad mental y mundos posibles. Los actos de la imaginación que dan sentido a la experiencia. Barcelona: Gedisa S.A.

Burgos García, Alicia, y Burgos Gómez, Elio Arturo. (2016). Estadística inferencial aplicada a la salud. Costa Rica: Cátedra Estadísticas para las Ciencias. Escuela de Ciencias Exactas y Naturales. UNED.

Burgos Gómez, Elio Arturo. (2016). Estadística descriptiva aplicada a las ciencias de la salud. Costa Rica: Cátedra Estadísticas para las Ciencias. Escuela de Ciencias Exactas y Naturales. UNED.

Canfux, Verónica. (2000). La pedagogía tradicional. En Tendencias pedagógicas en la realidad educativa actual (pp. 1-7). La Habana, Cuba: Universidad de la Habana. 
Chaves Esquivel, Edwin. (2000). Deserción y rendimiento académico en la UNED: 19961999. San José, Costa Rica: Centro de Investigación y Evaluación Institucional (CIEI). Universidad Estatal a Distancia.

Chaves Esquivel, Edwin. (2007). Una valoración sobre la enseñanza de la Estadística en los colegios académicos diurnos: regiones educativas de San José, Alajuela, Heredia, Pérez Zeledón y Upala. (Tesis para optar por el grado de Doctorado en Educación). Universidad Estatal a Distancia (UNED), San José, Costa Rica.

Comenio, Juan. (2002). Didáctica magna (12a. ed.). México: Porrúa.

Copleston, Frederick. (2004). Historia de la filosofía (Vol. 3). Barcelona: Ariel.

Daniel, Wayne W. (2008). Bioestadística: Base para el análisis de las ciencias de la salud (4a. ed.). México: Limusa Wiley.

Davýdov, Vasily Vasilovich. (1981). Tipos de generalización en la enseñanza. Cuba: Pueblo y Educación.

Del Valle, Roberto, y Fernández, Andrés. (2013). Desigualdad educativa en Costa Rica: la brecha entre estudiantes de colegios públicos y privados. Análisis con los resultados de la evaluación internacional pisa. Revista CEPAL, (111), 37-57.

Díez, José Antonio. (1997a). A hundred years of numbers. An historical introduction to measurement theory 1887-1990. Part I: The formation period. Two lines of research: axiomatics and real morphisms, scales and invariance. Stud. Hist. Phil. Sci., 28(1), 167185.

Díez, José Antonio. (1997b). A hundred years of numbers. An historical introduction to measurement theory 1887-1990. Part II: Suppes and the mature theory. Representation and uniqueness. Stud. Hist. Phil. Sci., 28(2), 237-265.

Díez, José Antonio. (2009). History of Measurement Theory. En History and Philosophy of Science and Technology. Encyclopedia of Life Support Systems (EOLSS). Oxford, UK: Developed under the Auspices of the UNESCO, Eolss Publishers. Recuperado de http://www.eolss.net

Dirección de Gestión y Evaluación de la Calidad. (2010). Primer informe sobre los resultados de la prueba para docentes de Matemática. Educación Secundaria. San José, Costa Rica: Ministerio de Educación Pública (MEP).

Dussel, Enrique. (1991). La producción teórica de Marx. Un comentario a los Grundrisse (2a. ed.). México: Siglo XXI editores. Recuperado de http://biblioteca.clacso.edu.ar/clacso/otros/20120424093754/Marx.pdf

Enrique Hevia, Frank Michel. (2016). Una concepción didáctica para el proceso de enseñanza-aprendizaje de la estadística en la formación inicial del profesor de matemática (Tesis presentada en opción al grado científico de Doctor en Ciencias 
Pedagógicas). Universidad de Ciencias Pedagógicas "Enrique José Varona", La Habana, Cuba.

Ertmer, Peggy A., y Newby, Timothy J. (2013). Behaviorism, cognitivism, constructivism: comparing critical features from an instructional design perspective. Performance Improvement Quarterly, 26(2), 43-71.

García del Valle, Alejandro. (1995). Apuntes de Estadística. Santiago, Chile: Tórculo Artes Gráficas, S.A.I.

Girón González-Torre, Francisco Javier. (1999). Determinismo, caos, azar e incertidumbre. En Horizontes culturales: las fronteras de la ciencia (pp. 73-83). España: Espasa Calpe.

Gómez Barrantes, Miguel. (2008). Elementos de estadística descriptiva (3a. ed.). San José. Costa Rica: EUNED.

Gómez Barrantes, Miguel. (2012). Elementos de estadística descriptiva (4a. ed.). San José. Costa Rica: EUNED.

Guerra, Mario, Correa, Juan, Núñez, Israel, y Scarón, Juan Miguel. (1994). Física. Elementos fundamentales. Mecánica y termodinámica clásicas. Relatividad Especial (Vol. I). Barcelona: Editorial REVERTË, S. A.

Hernández Rodríguez, Óscar. (2010). Elementos de probabilidades e inferencia estadística para Ciencias Sociales. San José, Costa Rica: Editorial de la Universidad de Costa Rica.

Hernández Rodríguez, Óscar. (2012). Estadística elemental para Ciencias Sociales (3a. ed.). San José, Costa Rica: Editorial de la Universidad de Costa Rica.

Herrera Obando, Lucía. (2005). Entrega de la docencia en cursos de alto nivel de dificultad. Informe de Investigación. San José, Costa Rica: Centro de Investigación y Evaluación Institucional (CIEI). Universidad Estatal a Distancia.

Iliénkov, Évald Vasílievich. (1975). Elevarse de lo abstracto a lo concreto. Publicado como E. Iliénkov «El Capital, teoría, estructura y método» (Tomo 1). México: Ediciones de Cultura Popular.

Iliénkov, Évald Vasílievich. (1977). Lógica dialéctica (J. Bayona, Trad.). Moscú, URSS: Progreso.

Irigoyen, Juan. (8 de agosto de 2013). El taylorismo educativo y sus víctimas. Recuperado de http://www.juanirigoyen.es/2013/08/el-taylorismo-educativo-y-susvictimas.html?spref=fb

Johnson, Robert. (1990). Estadística elemental. México, D. F.: Grupo Editorial Iberoamérica. 
Juan, Ángel A., de la Fuente, Blanca y Vila, Alicia. (2011). Estadística. Barcelona: Eureca Media, SL.

Juárez Duarte, José Alfredo, Ylé Martínez, Arturo, Flórez Arco, Armando, e Inzunsa Cázares, Santiago. (2012). Estadística: Exploración de datos (3a. ed.). México: Servicios Editoriales Once Ríos.

Kohan, Néstor. (2016). Marx y la lógica dialéctica en el capital [Video]. Buenos Aires: Brancaleone Films Cátedra Che Guevara. Recuperado de https://vimeo.com/298673809

Körner, Stephan. (1967). Introducción a la filosofía de la matemática. México: Siglo XXI editores.

Krantz, David H., Luce, Robert Duncan, Suppes, Patrick y Tversky, Amos. (2007). Foundations of measurement. Volume l: additive and polynomial representations (Vol. I). New York: Dover Publications, Inc.

Lefebvre, Henri. (1970). Lógica formal lógica dialéctica (M. E. Benítez Eiroa, Trad.). Madrid: Siglo XXI de España Editores.

Lind, Douglas A., Marshall, William G., y Wathen, Samuel A. (2012). Estadística aplicada a los negocios y la economía (15a. ed.). México: Mc Graw Hill.

Lipschutz, Seymour, y Schiller, John. (2000). Introducción a la probabilidad y estadística. Madrid: McGraw-Hill.

Luce, Robert Duncan (1996). The ongoing dialog between empirical science and measurement theory. Journal of Mathematical Psychology, 40, 78-98.

Luce, Robert Duncan, y Suppes, Patrick. (2002). Representational measurement theory. En Stanley Smith Stevens (ed.), Stevens' Handbook of Experimental Psychology (3a. ed., pp. 1-42). John Wiley \& Sons, Inc. Recuperado de http://media.wiley.com/product data/excerpt/87/04713788/0471378887.pdf

Marbán, Rocío M., y Pallecer, Julio A. (2002). Metrología para no-metrólogos (2a. ed.). Guatemala: OEA. Recuperado http://todometrologia.ucoz.com/mundometro/libros/metrolo all.pdf

Marx, Carlos. (1959). El capital. Crítica de la economía política (2a. ed., Vol. I). Bogotá, Colombia.

Mendenhall, William, Beaver, Robert J., y Beaver, Barbara M. (2010). Introducción a la probabilidad y estadística (13a. ed.). México: Cengage Learning Editores.

Ministerio de Educación Pública. (2012). Programa de estudio de Matemáticas. I y II Ciclo de la Educación Primaria, III Ciclo de Educación General Básica y Educación 
Diversificada. San José, Costa Rica. Recuperado de http://www.mep.go.cr/sites/default/files/programadeestudio/programas/matematica.pdf

Montanero Fernández, Jesús, y Minuesa Abril, Carmen. (2018). Estadística básica para para ciencias de la salud. Cáceres, España: Universidad de Extremadura, Servicio de Publicaciones.

Montero, Eiliana, Rojas, Shirley, Rodino, Ana María, y Zamora, Evelyn. (2012). Costa Rica en las pruebas PISA 2009 de competencia lectora y alfabetización matemática. Realizado para el Cuarto Informe del Estado de la Educación. San José, Costa Rica. Recuperado de

http://www.estadonacion.or.cr/files/biblioteca virtual/educacion/004/montero\%20et\%20 al pisa\%20competencia\%20lectora\%20y\%20alfabetizacion\%20matematica.pdf

Mora Rodríguez, Jesús. (3 de diciembre de 2013). Costa Rica entre los mejores de América Latina en las pruebas PISA, pero lejos de la élite [Noticias]. Recuperado de http://www.mep.go.cr/noticias/costa-rica-entre-mejores-america-latina-pruebas-pisapero-lejos-elite

Morettin, Luiz Gonzaga. (2010). Estatística básica: probabilidade e inferência, volume único. Sao Paulo: Pearson Prentice Hall.

Morin, Edgar. (2007). Los siete saberes necesarios para la educación del futuro. Buenos Aires: Nueva Visión.

Narens, Louis, y Luce, Robert Duncan. (1993). Further comments on the «non revolution» arising from axiomatic measurement theory. Psychological Science, 4(2), 127-130.

Pantoja Gutiérrez, Gabriel. (1984). Metodología de las ciencias sociales (Vol. I). México, D. F.: HARLA S. A.

Pérez Jacinto, Alipio Omar. (2006). Esquema conceptual referencial y operativo sobre los modelos estadísticos en las investigaciones educativas (Tesis en opción al grado científico de Doctor en Ciencias Pedagógicas). Instituto Superior Pedagógico «Enrique José Varona», Ciudad de la Habana, Cuba.

Pérez y Romero, J. Alejandro. (s. f.). Estadística y probabilidad: didácticamente para prepa. Universidad Autónoma de Querétaro. México.

Prigogine, llya. (1977). El Fin de las certidumbres (5a. ed.). Santiago, Chile: Editorial Andrés Bello.

Programa de lanzamiento de piedras. (2013). Programa de lanzamiento de piedras. Recuperado de http://web.educastur.princast.es/proyectos/azar/gravity.php

Quintana Ruiz, Carlos. (1983). Estadística elemental. San José. Costa Rica: Editorial de la Universidad de Costa Rica. 
Quintana Ruiz, Carlos. (1989). Elementos de inferencia estadística. San José. Costa Rica: Editorial de la Universidad de Costa Rica.

Quintana Valdés, Aurelio. (2010). Una estrategia didáctica para el proceso de enseñanzaaprendizaje del procesamiento de datos en la asignatura matemática en la educación de secundaria básica (Tesis presentada en Opción al grado Científico de Doctor en Ciencias Pedagógicas). Universidad de Ciencias Pedagógicas «Enrique José Varona», Ciudad de la Habana, Cuba.

Rao, Calyampudi Radhakrishna. (1997). Statistics and truth. Putting chance to work (2a. ed.). Singapore: World Scientific.

Reale, Giovanni. (1992). Introducción a Aristóteles (2a. ed., Vol. 16). Barcelona: Herder.

Reshetova, Zoya. A. (1988). Aplicación de los principios del enfoque sistémico en las asignaturas. En Análisis sistémico aplicado a la Educación Superior (pp. 85-97). La Habana, Cuba: CEPES-UC.

Rodríguez Ugidos, Zaira. (2001) Obras (2da. ed., Tomo 2). La Habana, Cuba: Editorial Pueblo y Educación.

Russell, Bertrand. (2009). Historia de la filosofía. España: Printer industria gráfica Newco, S.L.

Salama, David. (2002). Estadística: metodología y aplicaciones (5a. ed.). Caracas, Venezuela: Torino.

Salkind, Neil J. (1999). Métodos de Investigación (3a. ed.). México: Prentice Hall.

Salmina, N. G. Sorokin, V. V., y Chernysheva, V. K. (1984). Análisis lógico-psicológico de los procedimientos para construir la asignatura docente. La Educación Superior Contemporánea, 3(47), 55-78.

Spiegel, Murray R., y Stephen, Larry J. (2009). Estadística (4a. ed.). México, D. F.: McGrawHill/Interamericana Editores S.A.

Stevens, Stanley Smith (1946). On the theory of scales of measurment. Science, 103(2684), 677-680.

Stevens, Stanley Smith (1959). La medición y el hombre. (D. Leam, Trad.). México: Universidad Nacional de México.

Solano Castro, Adrián, y Umaña Carrillo, Roy. (2005). Estudio para incorporar elementos de la teoría de la actividad y la evaluación formativa, para el logro de aprendizajes significativos, en la enseñanza de la Estadística Aplicada (Avance de investigación). 
San José. Costa Rica: Centro de Mejoramiento de los Procesos Académicos (CEMPA). Universidad Estatal a Distancia (UNED).

Savage, C. Wade, y Ehrlich, Philip (1991). A brief introduction to measurement theory and to the essays. En Philosophical and foundational issues in measurement theory (p. 231). New Jersey: Lawrence Erlbaum Associates.

Triola, Mario. (2004). Estadística para las ciencias sociales (9a. ed.). México: Pearson Educación.

Triola, Mario. (2013). Estadística (11a. ed.). México: Pearson Educación.

Verdoy, Pablo Juan, Beltrán, Modesto Joaquín, y Peris, María José. (2015). Problemas resueltos de estadística aplicada a las ciencias sociales. España: Publicaciones de la Universitat Jaume I. Recuperado de www.sapientia.uji.es

Viertl, R. (2003). Foundations of statistics. En Encyclopedia of Life Support Systems (EOLSS), Developed under the Auspices of the UNESCO. Eolss Publishers, Oxford ,UK. Recuperado de http://www.eolss.net

Vygotski, Lev Semiónovich. (2000). El desarrollo de los procesos psicológicos superiores. Barcelona: Editorial Crítica.

Vygotsky, Lev Semiónovich. (1934). Pensamiento y lenguaje. Habana, Cuba: Editorial Pueblo y Educación.

Walpole, Ronald E., Myers, Raymond H., Myers, Sharon L., y Ye, Keying. (2012). Probabilidad y estadística para ingeniería y ciencias (9a. ed.). México: Pearson Educación.

Wilson, Jerry D., Buffa, Anthony J. y Lou, B. (2006). Física para las ciencias de la salud (6a. ed.). México: Pearson Educación.

Zeitlin, Irwing. (1986). Ideología y teoría sociológica. Buenos Aires: Amorrortu editores.

Zieffler, Andrew, y Catalysts for Change. (2013). Statistical thinking: A simulation approach to uncertainty (2a. ed.). Minneapolis, United States of America: MN: Catalyst Press. Recuperado a partir de http://zief0002.github.io/statistical-thinking/ 


\section{Revista indizada en}
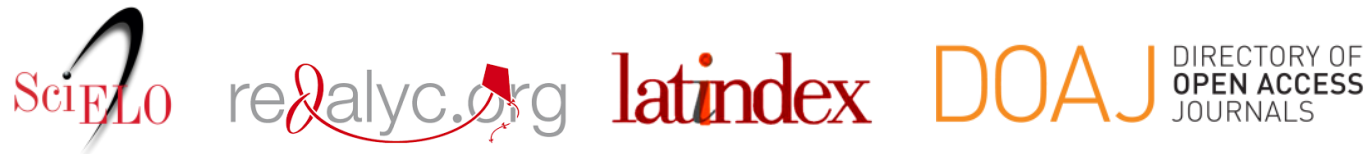

Distribuida en las bases de datos:

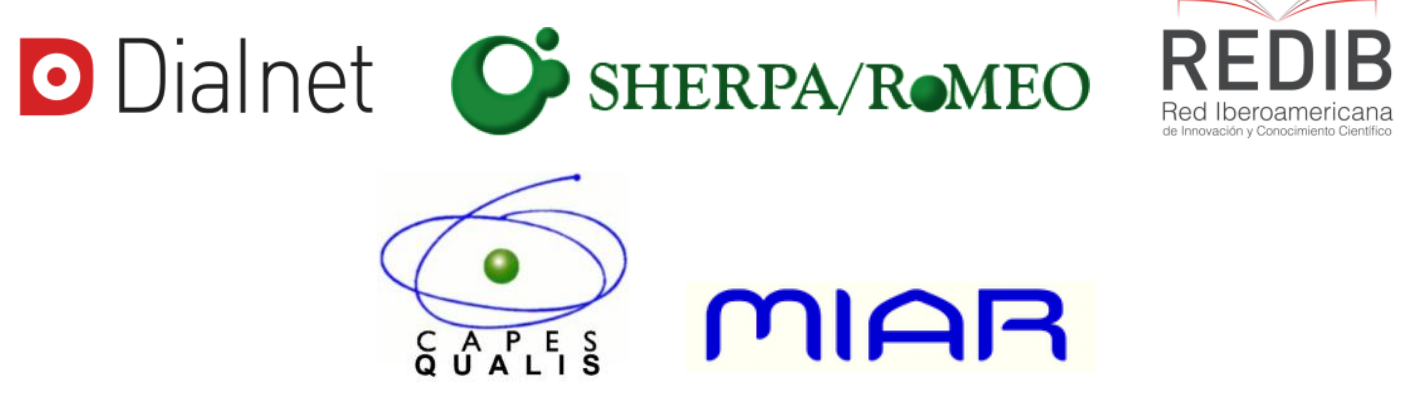

